\title{
Evaluation of the impact of EMBRACE II protocol in Spanish centers, with a large cohort of patients using a ranking index
}

\author{
Jose Chimeno, MSc', Naiara Fuentemilla, MSc², Paula Monasor, MSc³, Francisco Celada, MD!, Elena Villafranca, MD², \\ Silvia Rodriguez, MD³, María José Pérez-Calatayud, MD', Santiago Pellejero, PhD², Jose Pérez-Calatayud, PhDl,3 \\ IRadiotherapy Department, La Fe University Hospital, Valencia, Spain, ${ }^{2}$ Complejo Hospitalario de Navarra, Pamplona, Spain, ${ }^{3}$ Hospital Clinica \\ Benidorm, Benidorm, Spain
}

\begin{abstract}
Purpose: The aim of this paper was to assess development of high-dose-rate (HDR) cervix brachytherapy (BT) implants in three Spanish institutions before and after introduction of EMBRACE II protocol.

Material and methods: 392 patients from three different institutions, treated between 2009 and 2019 were analyzed. $\mathrm{D}_{90}$ of high-risk clinical target volume (HR-CTV) and $\mathrm{D}_{2 \mathrm{cc}}$ of organs at risk (OARs) of all patients were collected. Statistical distribution was analyzed for two different periods of time: before and after EMBRACE II publication. Index I was applied based on collected dosimetric quantities $\left(\mathrm{D}_{90}\right.$ and $\left.\mathrm{D}_{2 c c}\right)$ to enhance equilibrium between HR-CTV coverage and doses to OARs. Variation in dosimetry and index depending on CTV and technique used (IC vs. IC/IT) were also evaluated.

Results: Adaptation of institutions to EMBRACE II protocol resulted in a statistically significant increase of $\mathrm{D}_{90}$ HR-CTV (Institution 1; $p<0.00001$ ) or decrease of $\mathrm{D}_{2 c c}$ OARs (Institution 2; $p<0.04$ ). Increase in the use of interstitial component showed higher coverage of HR-CTV for Institution $3(p=0.03)$, and lower doses to OARs for the same coverage of HR-CTV at Institution $2(p$-OARs $<0.03)$. Even though index I was only significantly different between periods for Institution $1(p<0.0000001)$, it was able to show a reduction of dose variability related to higher expertise and higher interstitial component.

Conclusions: Depending on local protocol before EMBRACE II, the adaptation through increasing interstitial component and physician and physicist training, resulted in a significant increase of HR-CTV doses or reduction of OARs doses. Index I was able to describe an evolution of equilibrium between CTV coverage and OARs' sparing.

Key words: brachytherapy, cervix, dosimetric index, EMBRACE II.

\section{Purpose}

High-dose-rate (HDR) or pulsed-dose-rate (PDR) brachytherapy (BT) have demonstrated an essential role in the curative management of many pathologies, including cervical cancer. Excellent control rates of locally advanced cervical cancer (LACC) have been reported with external beam radiotherapy (EBRT) and concurrent chemotherapy, followed by BT [1, 2].

Technological evolution of EBRT and development of new techniques, such as intensity-modulated radiotherapy (IMRT), volumetric modulated arc therapy (VMAT), or stereotactic body radiotherapy (SBRT) have opened a new range of possible treatments for cervical cancer $[3,4]$. However, it has been reported that BT produces high-dose distributions in the target, with higher dose gradients delivered and better sparing of critical organs than EBRT $[5,6]$. The Society of Gynecologic Oncology (SGO), the Groupe Européen de Curiethérapie-European Society of Therapeutic Radiation and Oncology (GECESTRO), and the American Brachytherapy Society (ABS) in their recent recommendations state that the avoidance of BT for cervical cancer has been related to negative consequences affecting survival, and SBRT should not be a substitute for BT in patients undergoing primary curative intent radiation therapy for cervical cancer [5, 7].

Previously, with 2D BT treatment planning, protocols for cervix BT were based on dose points, such as points A or ICRU (International Commission on Radiation Units and Measurements) points [8-10]. Following introduction of computerized axial tomography (CT), the access to specific anatomy of patients turn out to be possible, and with 
dose-volume histograms (DVH) information, it became available for target volumes and organs at risk (OARs). However, protocols and tolerances were still based on dose points. In 2005-2006, GEC-ESTRO published recommendations for cervix cancer BT, in which T2-weighted magnetic resonance imaging (MRI) was the recommended image modality due to its' superior soft tissue contrast, which allowed identification of tumor response during treatment [11, 12]. These guidelines, later collected and supported by the ICRU Report No. 89 [13], introduced and defined a delineation procedure for gross tumor volume (GTV), high-risk clinical target volume (HR-CTV), intermediate-risk clinical target volume (IR-CTV), and OARs, focusing on dose prescriptions and tolerances of $90 \%$ and $2 \mathrm{~cm}^{3}$ of these volumes, respectively. Recommendations on dosimetric information, which should be reported included also: reference air-kerma rate (RAKR) of the source, dose to recto-vaginal point and points $A$, $\mathrm{D}_{98}$ (dose absorbed by $98 \%$ of the volume), $\mathrm{D}_{90}$, and $\mathrm{D}_{50}$ of HR-CTV, and $\mathrm{D}_{0.1 \mathrm{cc}}, \mathrm{D}_{2 \mathrm{cc}}$ of the bladder, rectum, sigmoid, and bowel (main OARs).

In 2008, GYN GEC-ESTRO workgroup started EMBRACE (IntErnational Study on MRI-based BRAchytherapy in CErvical Cancer), with a goal of assessing and validating MRI-guided BT. In this first study, dosimetric quantities introduced previously by GEC-ESTRO and later supported by ICRU (e.g., $\mathrm{D}_{90}, \mathrm{D}_{2 c c}$ ) were used, and their recommended values were based on historical and clinical experiences of participating institutions [14]. In 2011, a retrospective project (RetroEMBRACE) began with the aim of analyzing previously treated patients to beginning of EMBRACE [15]. With clinical data obtained from both the studies, the objectives of EMBRACE II study were established [16]. In this last protocol [17], the tolerance, objective, and mandatory values of different dosimetric parameters of each of relevant structures were reported, based on clinical evidence [18].

Recently, De Leeuw et al. [19] presented a report concerning a Dutch national quality assurance program for state-of-the-art curative radiotherapy for patients with LACC. This project was useful to show the need for training concerning the adaptation to the new EMBRACE II treatment protocol.

EMBRACE II protocol introduced new tolerance and objective doses for target and OARs volumes. The aim of the present paper was to analyze the evolution of dosimetric outcomes of cervix BT implants in three Spanish institutions, with respect to EMBRACE II publication.

\section{Material and methods}

Dosimetric and volumetric data of cervix BT interventions were collected. All data were obtained from three Spanish hospitals, with which the authors are affiliated. A total of 392 patients were analyzed, including 260 from Institution 1, 72 from Institution 2, and 56 from Institution 3. All patients followed a similar treatment scheme: EBRT followed by 4 MRI-based BT fractions applied in two applicator insertions, 7 Gy per fraction, prescribed according to a GEC-ESTRO biological spread sheet as a compromise between $\mathrm{D}_{90}$ to HR-CTV and IR-CTV, and
$\mathrm{D}_{2 c c}$ to OARs. EBRT component scheme varied according to a patient and Institution, and included 45 Gy in 25 fractions, 50.4 Gy in 28 fractions, 50 Gy in 25 fractions, 52.2 Gy in 29 fractions, 46 Gy in 23 fractions, 48.74 in 24 fractions, and 44.8 Gy in 28 fractions. Most common scheme was 45 Gy delivered in 25 fractions.

This study comprised patients from 2009 until 2019, depending on the data available at each Institution. For each patient, total dose and number of EBRT fractions, $\mathrm{D}_{90}$ of HR-CTV, and $\mathrm{D}_{2 \mathrm{cc}}$ of OARs of BT were obtained. In addition, HR-CTV volume (an average of two BT applications) and number of needles used were also recorded.

All three institutions used Elekta BT devices, such as Oncentra TPS (Elekta, Veenendaal, The Netherlands), Utrecht interstitial CT/MR applicator, and HDR-BT afterloader (one Institution had a MicroSelectron v.4, and the other two used Flexitron 1.0). Utrecht applicator combines intracavitary and interstitial gynecological BT. 4 and $6 \mathrm{~mm}$ diameter intra-uterine tubes, with 15 and 30 degrees curvatures were combined with 15, 20, and $25 \mathrm{~mm}$ ovoids, and up to five interstitial plastic needles in each ovoid, with three located in the outer side and two in the inner part, closer to the tandem [20]. Several patients were treated with tandem and ovoids only.

Even though the EMBRACE II protocol was published in 2018, certain institutions had access to the draft versions prior to that date. Not all hospitals could adapt their established protocols at the same time. Institution 1 adapted the EMBRACE II as soon as they obtained access to a draft version at the end of 2016. Institution 2, due to its' participation in the EMBRACE I clinical trial, could not completely adjust its' protocol until end of 2017. Institution 3 has still not completely changed the EMBRACE II objectives and constraints. Furthermore, Institution 3 included the sigmoid within delineation of the rectum structure. For this reason, it was assumed that $\mathrm{D}_{2 \mathrm{cc}}$ of the rectum and sigmoid were equal for all patients at this center.

Since the aim of the study was to assess the evolution of dosimetric quality of BT insertions, all dosimetric numbers were compared to the EMBRACE II objective/ tolerance levels for BT component. This protocol was used as a reference since it adds to the state of knowledge regarding local control of tumors and complications in OARs. Therefore, a dose in $\mathrm{EQD}_{2}$ (equivalent total doses in $2 \mathrm{~Gy}$ fractions computed using linear-quadratic model, with $\alpha / \beta=10 \mathrm{~Gy}$ for tumor and $\alpha / \beta=3$ Gy for OARs) of EBRT contribution was subtracted from objective/ tolerance values stated at EMBRACE II (Table 1) to obtain objective/tolerance values for BT. It was assumed that both $\mathrm{D}_{90}$ HR-CTV and $\mathrm{D}_{2 \mathrm{cc}}$ of OARs received EBRT prescribed dose.

The equilibrium between dose coverage of HR-CTV and dose of OARs was assessed using index I defined as:

$$
\begin{aligned}
& I=I_{C T V} \times I_{O A R} \\
& =\left(D_{90}^{\lim } / D_{90}^{o b j}-D_{90} / D_{90}^{o b j}\right) \times \\
& \times\left(D_{2 c c, \text { bladder }} / D_{2 c c, \text { bladder }}^{o b j} \times\left(D_{2 c c, \text { rectum }} / D_{2 c c, \text { rectum }}^{\text {obj }}\right) \times\right.
\end{aligned}
$$

where $D_{90}^{o b j}$ and $D_{2 c, i}^{o b j}$ are the objective doses (in EQD $\mathrm{E}_{2}$ ) for HR-CTV and tolerance levels for different OAR, and $D_{90}^{\lim }$ 
Table 1. Objective and tolerance prescription limits for HDR cervix treatment planning according to EMBRACE II protocol. EQD 2 includes $45 \mathrm{~Gy} / 25$ fractions of EBRT. Table taken from the EMBRACE II publication [16]

\begin{tabular}{|c|c|c|c|c|c|}
\hline Target & $\mathrm{D}_{90} \mathrm{HR}-\mathrm{CTV}$ EQD2 $2_{10}$ & $\mathrm{D}_{98} \mathrm{HR}-\mathrm{CTV}$ EQD2 ${ }_{10}$ & $\mathrm{D}_{98}$ GTV-res EQD2 10 & $\mathrm{D}_{98}$ IR-CTV EQD2 ${ }_{10}$ & Point A EQD2 $2_{10}$ \\
\hline Planning aims & $\begin{array}{l}>90 \mathrm{~Gy} \\
<95 \mathrm{~Gy}\end{array}$ & $>75 \mathrm{~Gy}$ & $>95 \mathrm{~Gy}$ & $>60 \mathrm{~Gy}$ & $>65 \mathrm{~Gy}$ \\
\hline $\begin{array}{l}\text { Limits for pre- } \\
\text { scribed dose }\end{array}$ & $<85$ Gy & - & $>90$ Gy & - & - \\
\hline OARs & Bladder $D_{2 c c} E Q D 2_{3}$ & Rectum $D_{2 c c} E Q D 2_{3}$ & $\begin{array}{c}\text { Recto-vaginal point } \\
\text { EQD2 }\end{array}$ & Sigmoid $D_{2 c c} E Q D 2_{3}$ & Bowel $D_{2 c c} E Q D 2_{3}$ \\
\hline Planning aims & $<80 \mathrm{~Gy}$ & $<65$ Gy & $<65$ Gy & $<70 \mathrm{~Gy}^{*}$ & $<70 \mathrm{~Gy}^{*}$ \\
\hline $\begin{array}{l}\text { Limits for pre- } \\
\text { scribed dose }\end{array}$ & $<90$ Gy & $<75$ Gy & $<75$ Gy & $<75 \mathrm{~Gy}^{\star}$ & $<75 \mathrm{~Gy}^{\star}$ \\
\hline
\end{tabular}

* For the sigmoid/bowel structures, this dose constrains are valid in case of non-mobile bowel loops, resulting in a situation where the most exposed volume is located at a similar part of the organ

Table 2. Summary of patients' cohort. Volume of HR-CTV represents mean between two applications. All uncertainties represent $\mathrm{k}=1$

\begin{tabular}{cccccccccc} 
& \multicolumn{4}{c}{ Pre-EMBRACE II } & \multicolumn{4}{c}{ Post-EMBRACE II } \\
\cline { 2 - 10 } & $n$ & $\begin{array}{c}\text { HR-CTV } \\
\text { volume }\left(\mathrm{cm}^{3}\right)\end{array}$ & IC (\%) & IC/IT (\%) & $n$ & $\begin{array}{c}\text { HR-CTV } \\
\text { volume }\left(\mathrm{cm}^{3}\right)\end{array}$ & IC (\%) & IC/IT (\%) \\
\hline 1 & 121 & $21 \pm 13$ & 3 & 97 & 139 & $17 \pm 10$ & 2 & 98 \\
\hline 2 & 46 & $28 \pm 19$ & 66 & 34 & 25 & $23 \pm 17$ & 24 & 76 \\
\hline 3 & 56 & $62 \pm 14$ & 43 & 57 & - & - & - & -
\end{tabular}

is the upper limit dose for HR-CTV. It should be noted that this objective and tolerance doses refer only to BT component. For example, if EBRT treatment consisted of 45 Gy with $1.8 \mathrm{~Gy} /$ fraction (44.25 Gy EQD2 ${ }_{10}$ and $43.2 \mathrm{~Gy}$ $\mathrm{EQD2} 2), D_{90}^{\text {obj }}=90-44.25=45.75$ Gy EQD2 $10, D_{90}^{\lim }=$ $95-44.25=50.75$ Gy EQD2 10 , and $D_{2 c c, b l a d d e r}^{o b j}=80-43.2=$ 36.8 Gy EQD2 3 . According to this definition, the optimal value of index I was 0 . There is a critical case, in which $\mathrm{D}_{90}$ is equal to $D_{90}^{\lim }$, which causes the value of index to be independent of OARs doses. However, with a sufficient decimal precision, this should not occur. In all treatments with $\mathrm{D}_{90}$ higher than $D_{90}^{\lim }$, the index had a negative value. Moreover, the proportion with OARs was kept with-

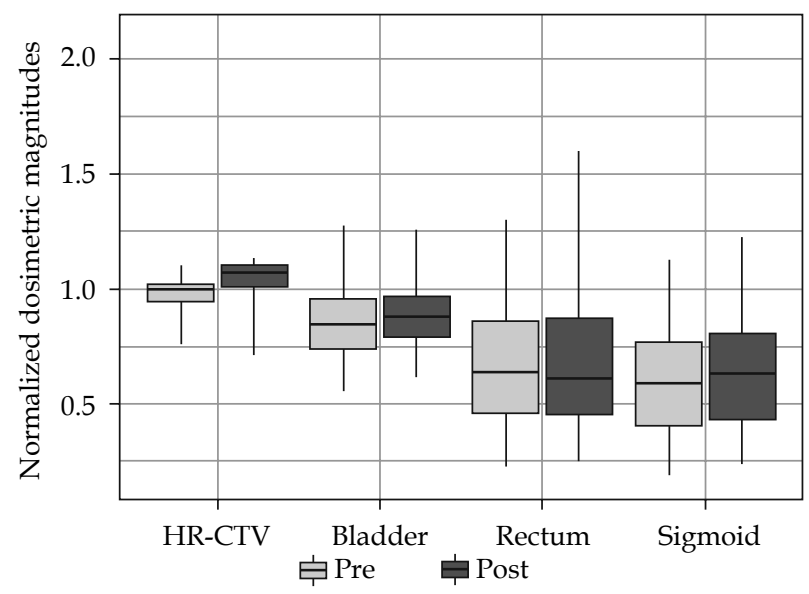

Fig. 1. Values of $D_{90}$ and $D_{2 c c}$ of HR-CTV and OARs of Institution 1 for pre- and post-period of adaptation to EMBRACE II in negative range, and treatments with a higher absolute value had a worse assessment. $D_{90}^{o b j}$ has to be understood as a minimum objective value. Since EMBRACE II established a planning aim of $\mathrm{D}_{90}$ between 90 Gy EQD2 10 and 95 Gy EQD2 $2_{10}$, higher doses within this interval were considered in the present study. Consequently, index I reached its' optimal value, when $D_{90}$ equals to $D_{90}^{\lim }$, and not when corresponds to $D_{90}^{o b j}$. None of the patients presented with $\mathrm{D}_{90}$ of HR-CTV lower than 85 Gy EQD2 10 .

\section{Statistical analysis}

Statistical analysis of all the dosimetric results was carried out using RStudio software version 1.2.1335. RStudio is an environment for $\mathrm{R}$, a programming language for statistical computing. For those institutions, which have adapted to the EMBRACE II protocol, assessment of statistically relevant differences between two periods (i.e., pre- and post-EMBRACE II) was performed. Depending on distribution of characteristics, Welch, Wilcoxon, or Yuen statistical tests were applied. A $p$-value of $<0.05$ was considered statistically significant.

\section{Results}

Table 2 presents a summary of the analyzed cohort, and a percentage of pure IC and IC/ IT techniques in both periods.

Figures 1-3 summarize the values and evolution of $\mathrm{D}_{90}$ of HR-CTV, and $\mathrm{D}_{2 \mathrm{cc}}$ of OARs for each Institution, normalized by objective or tolerance value for brachytherapy component of the treatment. This means, for example, for a patient who received 43.2 Gy EQD2 ${ }_{3}$ in EBRT, normal- 


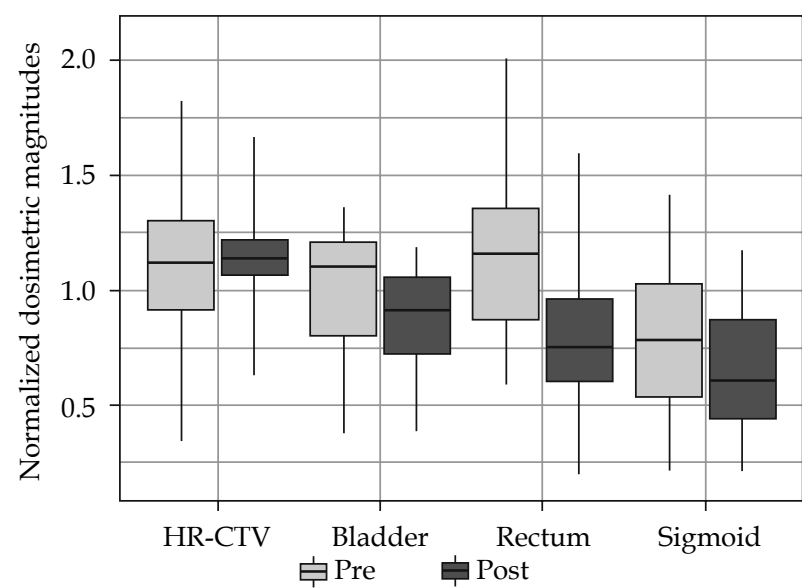

Fig. 2. Values of $D_{90}$ and $D_{2 c c}$ of HR-CTV and OARs of Institution 2 for pre- and post-period of adaptation to EMBRACE II

ization factor of $\mathrm{D}_{2 \mathrm{cc}}$ rectum would be $21.8 \mathrm{~Gy}$ EQD2 3 . However, for the same patient, normalization factor of $\mathrm{D}_{2 \mathrm{cc}}$ sigmoid would be $26.8 \mathrm{~Gy} \mathrm{EQD2} 2_{3}$. In any case, quantities plotted in Figures 1-3 are equal to one, when HRCTV receives exactly $90 \mathrm{~Gy}_{10}$ in HR-CTV, $80 \mathrm{~Gy}_{3}$ in the bladder, $65 \mathrm{~Gy}_{3}$ in the rectum, and $70 \mathrm{~Gy}_{3}$ in the sigmoid after the overall treatment. Boxes in these figures represent the $25^{\text {th }}$ and $75^{\text {th }}$ percentiles, horizontal line shows the median, and extremes of vertical lines, with $95 \%$ of distribution.

As can be seen in Figure 1, after adapting of EMBRACE II in Institution 1, a conservative approach, increasing the dose to HR-CTV enough to be able to keep OARs almost unchanged, reached a median value of 1.071 for normalized $D_{90}$. In fact, only $D_{90}$ was found to present a statistical difference between both periods $(p=0.000003)$. On the other hand, Institution 2 already approached a high-dose in HR-CTV, and even exceeded in some cases 96 Gy and 95 Gy thresholds established in EMBRACE I and EMBRACE II, respectively. The adap-

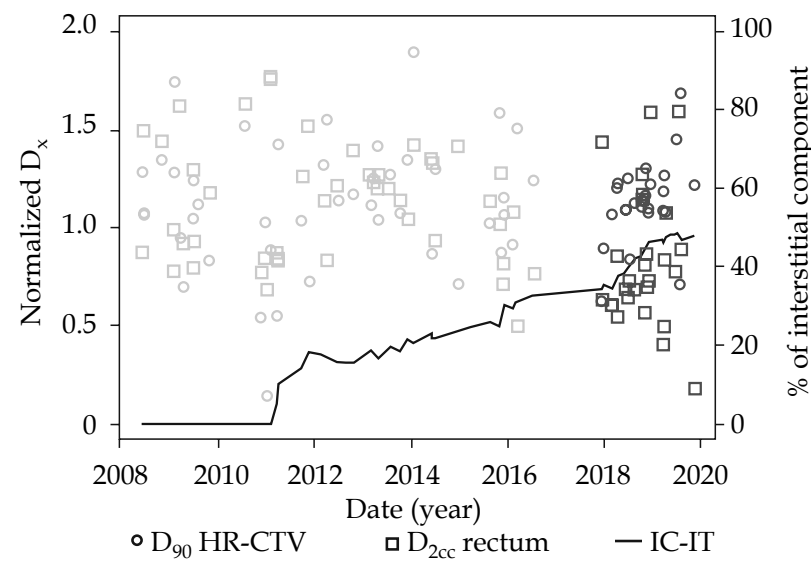

Fig. 4. Evolution of $D_{90} H R-C T V$ and $D_{2 c c}$ of the rectum in Institution 2, together with the percentage of interstitial implants during the whole analyzed period. Blue and red markers represent treatments during pre- and postEMBRACE II publication

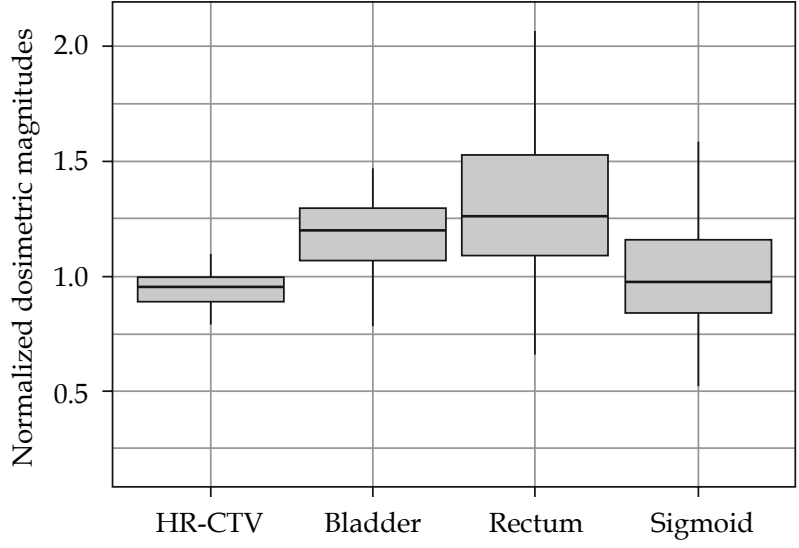

Fig. 3. Values of $D_{90}$ and $D_{2 c c}$ of HR-CTV and OARs of Institution 3

tation to new constraints was translated to a statistically significant reduction of doses to OARs $\left(p_{\text {bladder }}=0.03, p_{\text {rec- }}\right.$ tum $=0.0004, p_{\text {sigmoid }}=0.02$ ), and delivery of more concentrated HR-CTV dose between objective and upper threshold, as shown in a smaller box in Figure 2. Figure 4 shows an evolution of $D_{90}$ HR-CTV and $D_{2 c c}$ of the rectum in Institution 2, together with a percentage of interstitial implants with respect to the total number of patients. Since the tolerance limits of OARs were less demanding in the EMBRACE I protocol, distributions of OARs in Figure 3 for Institution 3 were mostly above unity, and distribution of $\mathrm{D}_{90}$ of HR-CTV was below unity.

Index I introduced the above evaluates, with an effect of newly established EMBRACE II objectives having worse values for those treatments with a higher absolute value. Figure 5 and Table 3 represent the distribution of index I for the three centers. A tendency towards higher HR-CTV doses in Institution 2 (Figure 2) was interpreted here as a significant percentage of index I distribution being negative. High doses to OARs (with respect to the

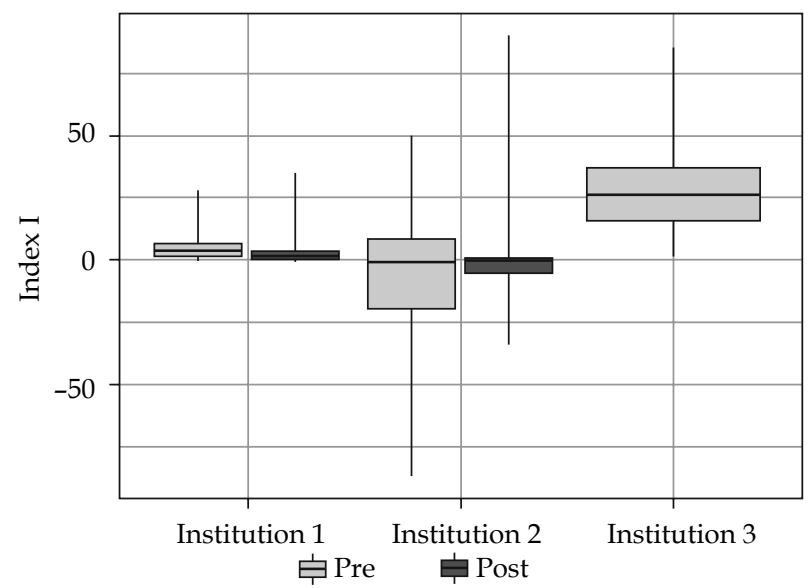

Fig. 5. Index I of the three Institutions in the two periods analyzed. Box represents the $25^{\text {th }}$ and $75^{\text {th }}$ percentiles and extremes of vertical lines, including $95 \%$ of distribution 
Table 3. Summary of index I distribution for all three centers with two analyzed periods. $P$-value between analyzed periods is included with significance for values lower than 0.05

\begin{tabular}{lccccccc} 
Institution & \multicolumn{7}{c}{ Pre-EMBRACE II } \\
\cline { 2 - 9 } & Mean & SD & Range & Mean & SD & Range & $p$-value \\
\hline 1 & 6 & 7 & $-0.1-46.9$ & 4 & 8 & $-3.1-65.8$ & 0.0000001 \\
\hline 2 & -5 & 28 & $-110.8-59.0$ & 2 & 22 & $-34.1-90.3$ & 0.06 \\
\hline 3 & 28 & 19 & $1.8-113.9$ & - & - & - & -
\end{tabular}

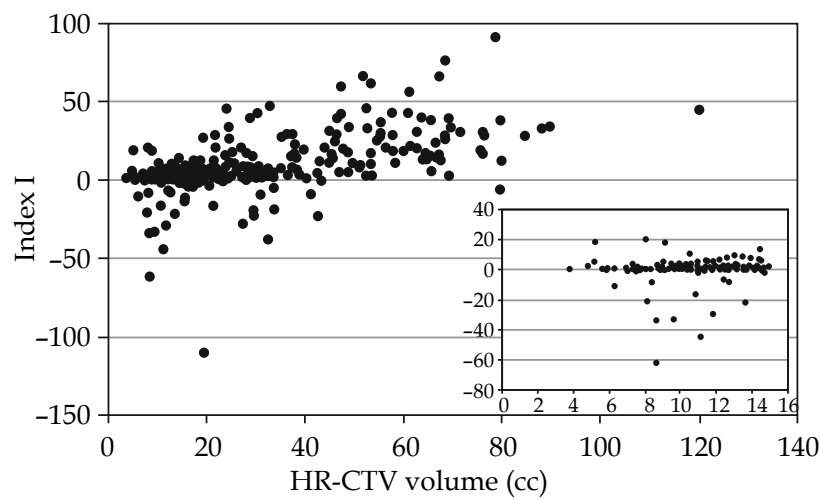

Fig. 6. Dependence of index I with the volume of HR-CTV. Inner graph zooms out the region on volumes lower than $15 \mathrm{~cm}^{3}$

EMBRACE II constraints) of implants in Institution 3 were expressed by index I with higher values. Index I was significantly different only for Institution $1(p=0.0000001)$ for the two analyzed periods. Even though the median was not statistically different for Institution $2(p=0.06)$, the index I integrated the reduction of variation shown in Figure 2 for both target volume and OARs. Quality of an implant depends on many factors, especially volume of the CTV. The volume of HR-CTV influences the level of conformity and doses to OARs, so that it is possible to achieve without an interstitial component. As can be seen in Figure 6, the equilibrium between $D_{90}$ of HR-CTV and $\mathrm{D}_{2 \mathrm{cc}}$ of OARs was harder to handle for larger CTV volumes, which was expressed by an increase of index I with volume of the tumor.

\section{Discussion}

This study was a retrospective analysis, in which dosimetric information used did not affect patients' treatments. A total of 392 patients from three Spanish centers, with higher number of cervix BT treatments were analyzed. Most patients were treated from 2012 to 2019. This was a period of numerous developments and published recommendations by international societies. The hypothesis tested was to assess the improvement of both implants and dosimetry planning with time, based on training and experience of both physicians and physicists. The analysis presented in this study showed three different approaches to the above-mentioned adaptation that depended on available resources, adaptation of clinical and optimization protocols, and characteristics of the treated patients among other factors. Institution 1 from the beginning, treated almost all patients with an intersti- tial component. In this case, the goal was to increase the dose to the tumor while keeping OARs at least as good as in the period of EMBRACE I. Institution 2 evolved from a majority of pure intracavitary implants. The main impact of this change was a statistically significant reduction of doses to OARs depending on the technique used $\left(\mathrm{IC}\right.$ vs. $\left.\mathrm{IC} / \mathrm{IT} ; p_{\text {bladder }}=0.02, p_{\text {rectum }}=0.003, p_{\text {sigmoid }}=0.03\right)$. Institution 2 sought a maximum allowed dose to CTV, even overreaching this threshold in some patients. The peculiarity of large tumor volume of patients in Institution 3 , and the majority of intracavitary implants (Table 2) led to a subtle evolution and difficult equilibrium between $D_{90}$ (coverage of tumor) and OARs. The lack of sufficient interstitial components in an implant of aboveaverage HR-CTV volume, could lead to increased tandem loading, resulting in a more cylindrical dose distribution and increased dose to OARs. As can be expected, those implants with interstitial component led to higher coverage and at least equal OARs sparing $\left(p_{\mathrm{HR}-\mathrm{CTV}}=0.03\right)$. This effect was mainly caused by better management of OARs doses than the interstitial component could provide [20, 21]. In Figure 6, treatments with higher HR-CTV volumes were ranked lower by index I. This was in fact due to the above-mentioned effect and lack of HR-CTV coverage resulting from OARs limitations [22].

Although all three institutions used MRI for contouring purposes, the distribution of HR-CTV volumes was different between centers (Table 2). This difference may be related to different patient referral patterns as well as specific geographical and screening strategies.

The distribution of HR-CTV volumes published varied between studies. The EMBRACE group has published some studies, which included HR-CTV volume statistics. Jastaniyah et al. [23] presented a volumetric analysis of $\mathrm{GTV}_{\mathrm{D}}$ (gross tumor volume at diagnosis) and HR-CTV defined according to GEC ESTRO recommendations. In this case, the reported mean and standard deviation was 31.6 $\pm 16.1 \mathrm{~cm}^{3}$. Recently, the EMBRACE I group [24] reported that HR-CTV mean volume of 1,416 patients was $28 \mathrm{~cm}^{3}$, with $95 \%$ confidence interval $\left(20-40 \mathrm{~cm}^{3}\right)$. Also, in Figure 1 , an estimated range of $8-117 \mathrm{~cm}^{3}$ is presented [17].

Other studies with HR-CTV volume statistical data have been also published. Cannon et al. showed [25] their results of 2020 audit, in which mean HR-CTV volume was $19.27 \mathrm{~cm}^{3}$ (range, 9.9-29.33 $\mathrm{cm}^{3}$ ), and Oud et al. [26] reported HR-CTV average volume of 34 patients as 29.4 $\pm 12.5 \mathrm{~cm}^{3}$ (range, $8.5-92 \mathrm{~cm}^{3}$ ).

A limitation of our study, aiming at index evaluation, was a lack of contouring uncertainty analysis. In Institutions 2 and 3, a single radiation oncologist performs BT treatments, which confirms the absence of inter-observer 
variation; on the other hand, in center 1 , two radiation oncologists carry out the contouring.

All institutions had a learning and skill curve with regard to the application of interstitial component. Initially, few needles were used, but over time, more and deeper needles were employed not only for enhancing dose conformity to HR-CTV, but also for sparing of OARs. The trend shown in Figure 4 represents this evolution. In Institution 2, significantly lower doses were applied to all OARs when an interstitial component was used $\left(p_{\text {bladder }}\right.$ $=0.02, p_{\text {rectum }}=0.003, p_{\text {sigmoid }}=0.03, p_{\text {HR-CTV }}=0.3$ ). For Institution 3, the opposite results were found; only the coverage of the target volume was significantly different when needles were included $\left(p_{\text {bladder }}=0.8, p_{\text {rectum }}=0.3\right.$, $p_{\text {sigmoid }}=0.3, p_{\mathrm{HR}-\mathrm{CTV}}=0.03$ ). In order to understand the difference in OARs doses between Institutions and for each institution, two relevant points must be considered. On the one hand, threshold doses for OARs present significant variations between EMBRACE I and EMBRACE II projects, since the former limits were based on historical experience of only a few institutions, and the latter were supported by clinical evidence. Alternatively, the adaptation of each center's protocol to new guidelines of the EMBRACE II project was not initiated at the same time. New objectives for HR-CTV coverage and OARs sparing could also mean adapting the optimization process and/ or the necessity to include more interstitial components in learning process to induce changes.

Index I was used as a metric to illustrate the impact of EMBRACE II in dosimetric outcomes of HDR-BT implants. This index endeavors to assess the equilibrium between HR-CTV coverage and OARs sparing, but not to assess individual plans. However, some limitations must be outlined. There was a critical point in the definition. In those cases, in which $D_{90}$ equals to $D_{90}{ }^{l i m}$, the index equals to 0 , independently of OARs doses. This situation was always avoided using decimal precision of the treatment planning system (TPS) and Excel sheets applied for radiobiological calculations. This critical point would make the index non-applicable for institutions or specific patients, where treatment was re-normalized in order to equal $D_{90}$ to $D_{90}$ lim. It is interesting to analyze individually both terms, i.e., $\mathrm{I}_{\mathrm{CTV}}$ and $\mathrm{I}_{\mathrm{OAR}}$, as compensation between these two components could occur. However, such compensation was uncommon, since only sub-optimal implants (rare anatomy, lack of interstitial component, etc.) led to low doses and poor coverage of HR-CTV, high OARs doses, or high doses to both, target volume and OARs.

\section{Conclusions}

Embrace II and ICRU 89 introduced a new paradigm on BT planning towards higher doses to target volumes and OARs better sparing. In order to achieve these new objectives, an increase of interstitial component is needed. However, not all centers had the training or protocol flexibility to adapt to these new guidelines. A total of 392 patients from three Spanish institutions, with a majority of cervix brachytherapy treatments were analyzed. Depending on the local protocol before EMBRACE II, the adaptation was achieved by increasing the interstitial component and the physician and physicist training, which resulted in a significant increase of HR-CTV doses or a reduction of OARs doses. The introduced index I has been able to quantify the evolution of the equilibrium between CTV coverage and OARs sparing, according to EMBRACE II objectives and constraints.

\section{Disclosure}

The authors report no conflict of interest.

\section{References}

1. Nag S, Erickson B, Thomadsen B et al. The American Brachytherapy Society recommendations for high-dose-rate brachytherapy for carcinoma of the cervix. Int J Radiat Oncol Biol Phys 2000; 48: 201-211.

2. Han K, Milosevic M, Fyles A et al. Trends in the utilization of brachytherapy in cervical cancer in the United States. Int J Radiat Oncol Biol Phys 2013; 87: 111-119.

3. Kemmerer E, Hernandez E, Ferriss JS et al. Use of image-guided stereotactic body radiation therapy in lieu of intracavitary brachytherapy for the treatment of inoperable endometrial neoplasia. Int J Radiat Oncol Biol Phys 2013; 85: 129-135.

4. Jones R, Chen Q, Best R et al. Dosimetric feasibility of stereotactic body radiation therapy as an alternative to brachytherapy for definitive treatment of medically inoperable early stage endometrial cancer. Radiat Oncol 2014; 9: 164.

5. Tanderup K, Eifel P, Yashar C et al. Curative radiation therapy for locally advanced cervical cancer: brachytherapy is NOT optional. Int J Radiat Oncol Biol Phys 2014; 88: 537-539.

6. Tanderup K, Lindegaard JC, Kirisits C et al. Image Guided Adaptative Brachytherapy in cervix cancer: A new paradigm changing clinical practice and outcome. Radiother Oncol 2016; 120: $365-369$

7. Holschneider $\mathrm{CH}$, Petereit DG, Chu C et al. Brachytherapy: A critical component of primary radiation therapy for cervical cancer: From the Society of Gynecologic Oncology (SGO) and the American Brachytherapy Society (ABS). Gynecol Oncol 2019; 152: 540-547.

8. Chassagne D, Dutreix A, Almond P et al. Dose and volume specification for reporting intracavitary therapy in gynecology. Report 38. J ICRU 1985; 20: 1-21.

9. Viswanathan AN, Thomadsen B. American Brachytherapy Society consensus guidelines for locally advanced carcinoma of the cervix. Part I: General principles. Brachytherapy 2012; 11: 33-46.

10. Viswanathan AN, Beriwal S, De Los Santos JF et al. American Brachytherapy Society consensus guidelines for locally advanced carcinoma of the cervix. Part II: High-dose-rate brachytherapy. Brachytherapy 2012; 11: 47-52.

11. Haie-Meder C, Potter R, Van Limbergen E et al. Recommendations from gynaecologial (GYN) GEC ESTRO working group (I): concepts and terms in 3D image-based 3D treatment planning in cervix cancer brachytherapy with emphasis on MRI assessment of GTV and CTV. Radiother Oncol 2005; 74: 235-245.

12. Potter R, Haie-Meder C, Van Limbergen E et al. Recommendations from gynecological (GYN) GEC ESTRO working group (II): concepts and terms in 3D mage-based treatment planning in cervix cancer brachytherapy-3D dose volume parameters and aspects of 3D image-based anatomy, radiation physics, radiobiology. Radiother Oncol 2006; 78: 67-77.

13. International Commission on Radiation Units and Measurements. ICRU report 89: Prescribing, recording, and reporting brachytherapy for Cancer of cervix. J ICRU 2016; 13. 
14. International study on MRI-guided brachytherapy in locally advanced cervical cancer. University of Vienna; https:// www.embracestudy.dk

15. Retro-embrace. Medical University of Vienna; https://www. retroembrace.com

16. EMBRACE II study protocol image guided intensity modulated external beam radiochemotherapy and MRI based adaptative brachytherapy in locally advanced cervical cancer; https://www.embracestudy.dk

17. Pötter R, Tanderup K, Kirisits $C$ et al. The EMBRACE II study: The outcome and prospect of two decades of evolution within the GEC-ESTRO GYN working group and the EMBRACE studies. Clin Transl Radiat Oncol 2018; 9: 48-60.

18. Sturdza A, Pötter R, Fokdal LU et al. Image guided brachytherapy in locally advanced cervical cancer: improved pelvic control and survival in RetroEMBRACE, a multicenter cohort study. Radiother Oncol 2016; 120: 428-433.

19. De Leeuw A, Nout RA, Van Leeuwen R et al. Implementation of state-of-the-art (chemo)radiation for advanced cervix cancer in the Netherlands: A quality improvement program. Tech Innov Patient Support Radiat Oncol 2019; 9: 1-7.

20. Serban M, Kirisits C, De Leeuw A et al. (the EMBRACE Collaborative Group). Ring versus ovoids and intracavitary versus intracavitary-interstitial applicators in cervical cancer brachytherapy: Results from the EMBRACE I study. Int $\mathrm{J} R \mathrm{a}-$ diat Oncol Biol Phys 2020; 106: 1052-1062.

21. Nomden CN, de Leeuw AAC, Van Limbergen E et al. Multicentre treatment planning study of MRI-guided brachytherapy for cervical cancer: Comparison between tandem-ovoid applicator users. Radiother Oncol 2013; 107: 82-87.

22. Nomden CN, De Leeuw Astrid AC, Moerland MA et al. Clinical use of the Utrecht applicator for combined intracavitary/ interstitial brachytherapy treatment in locally advanced cervical cancer. Int J Radiat Oncol Biol Phys 2012; 82: 1424-1430.

23. Jastaniyah N, Yoshida $\mathrm{K}$, Tanderup $\mathrm{K}$ et al. A volumetric analysis of GTVD and CTVHR as defined by GEC ESTRO recommendations in FIGO stage IIB and IIIB cervical cancer patients treated with IGABT in a prospective multicentric trial (EMBRACE). Radiother Oncol 2016; 120: 404-411.

24. Pötter R, Tanderup K, Schmid MP et al. MRI-guided adaptive brachytherapy in locally advanced cervical cancer (EMBRACE-I): a multicentre prospective cohort study. Lancet Oncol 2021; 22: 538-547.

25. Cannon J, Bownes P, Mason J, Cooper R. UK audit of target volume and organ at risk delineation and dose optimisation for cervix radiotherapy treatments. Br J Radiol 2020; 93: 20190897.

26. Oud M, Kolkman-Deurloo IK, Mens JW et al. Fast and fullyautomated multi-criterial treatment planning for adaptive HDR brachytherapy for locally advanced cervical cancer. Radiother Oncol 2020; 148: 143-150. 